\title{
A nomogram to predict vascular invasion before resection of colorectal cancer
}

\author{
WEISHUN XIE ${ }^{1,2^{*}}$, JUNGANG LIU ${ }^{1,2^{*}}$, XIAOLIANG HUANG ${ }^{1,2}$, GUO WU $^{1,2}$, \\ FRANCO JEEN $^{1,2}$, SHAOMEI CHEN ${ }^{1,2}$, CHUQIAO ZHANG ${ }^{1,2}$, WENKANG YANG ${ }^{1,2}$, \\ CHAN LI ${ }^{1}$, ZHENGTIAN LI $^{1,2}$, LIANYING GE ${ }^{2,3}$ and WEIZHONG TANG ${ }^{1,2}$ \\ ${ }^{1}$ Department of Gastrointestinal Surgery; ${ }^{2}$ Guangxi Clinical Research Center for Colorectal Cancer; \\ ${ }^{3}$ Department of Gynecologic Oncology, Affiliated Tumor Hospital of Guangxi Medical University, \\ Nanning, Guangxi 530021, P.R. China
}

Received January 18, 2019; Accepted July 26, 2019

DOI: 10.3892/ol.2019.10937

\begin{abstract}
Vascular invasion (VI) is an important feature for systemic recurrence and an indicator for the application of adjuvant therapy in colorectal cancer (CRC). Preoperative knowledge of VI is important in determining whether adjuvant therapy is necessary, as well as the adequacy of surgical resection. In the present study, a predictive nomogram for VI in patients with CRC was constructed. The prediction model consisted of 664 eligible patients with CRC, who were divided into a training set $(n=468)$ and a validation set $(n=196)$. Data were collected between August 2013 and April 2018. The feature selection model was established using the least absolute shrinkage and selection operator regression model. Multivariable logistic regression analysis was used to construct the predictive nomogram. The performance of the nomogram was evaluated by calibration, discrimination and clinical usefulness. Differentiation, computed tomography (CT)-based on $\mathrm{N}$ stage (CT N stage), hemameba and tumor distance from the anus $(\mathrm{cm})$ were integrated into the nomogram. The nomogram exhibited good discrimination, with an area under the curve (AUC) of 0.731 and good calibration. Application of the nomogram in the validation cohort showed acceptable discrimination, with an AUC of 0.710 and good calibration. Decision curve analysis revealed that the nomogram was clinically useful. These findings suggests, to the best of our knowledge, that this may be the first nomogram for individual
\end{abstract}

Correspondence to: Professor Lianying Ge or Professor Weizhong Tang, Department of Gynecologic Oncology, 71 Hedi Road, Affiliated Tumor Hospital of Guangxi Medical University, Nanning, Guangxi 530021, P.R. China

E-mail: gelianying733@163.com

E-mail: tangweizhong@gxmu.edu.cn

*Contributed equally

Key words: nomogram, vascular invasion, colorectal cancer, prediction model preoperative prediction of VI in patients with CRC, which may promote preoperative optimization strategies for this selected group of patients.

\section{Introduction}

According to the 2018 global cancer statistics, colorectal cancer (CRC) has the third highest incidence rate of all types of cancers worldwide; it also has the second highest mortality rate (1). In USA, it has been estimated that there will be $>140,000$ new CRC cases and 23,380 deaths 2019, accounting for $\sim 1$ in 7 cancer cases and deaths (2).

Adjuvant therapy is typically selected by pathological and clinical staging as two of the prognostic predictors (3). Vascular invasion (VI) is prevalent in CRC; it has been reported that $23 \%$ of CRC and VI cases are combined in postoperative pathology (4). Therefore, accurate identification of VI involvement in patients with CRC is crucial for prognosis and treatment strategy decisions.

VI is a strong prognostic indicator in CRC (3). A previous study has shown that there is a significant association between VI and metastasis and a high rate of recurrence in univariate analysis and multivariable analysis (5). In the Union for International Cancer Control, stage I rectal cancer (T1, T2 N0), VI is a high-risk factor for disease progression and recurrence (6). In addition, VI is associated with poor loco-regional outcomes, which are potentially valuable predictors of local recurrence in rectal cancers (7). A previous study demonstrated that VI is also associated with reduced overall survival rate and disease-free survival rates, therefore serving as a strong prognostic marker (8). In the clinical practice guidelines published by the National Comprehensive Cancer Network (NCCN) (3), VI is regarded as a high-risk factor for systemic recurrence and stage III or high-risk stage II.

It is recommended that patients with $\mathrm{CRC}$ with VI receive adjuvant therapy (9). VI is a strong prognostic indicator, and a predictor of CRC metastasis and recurrence (5). A better understanding of VI supports the decision of whether adjuvant therapy is required, determining the adequacy of surgical resection, and selecting the optimal treatment (8). Vascular endothelial growth factor (VEGF) and its expressed 
products Fms related tyrosine kinase-1 and kinase insert domain receptor, regulate endothelial cell proliferation, migration, invasion, survival and branching morphogenesis, which are reported to be associated with VI $(10,11)$. However, the practicality of these biomarkers is limited $(10,11)$.

Since the first reported clinical application of a nomogram in 1928 (12), nomograms have attracted increased attention. A nomogram is a user-friendly graphical prediction model with strong clinical application (13-15). Users can build the nomogram to obtain points assigned to each predicted factor at the top of the scale (13-15). Through this, the total points can be transformed to predict the possible risk of a specific event for patients in the lowest scale. To date, nomograms have been widely used in the diagnosis and prognostic prediction of a variety of malignancies, such as Ewing sarcoma and thymoma prognosis $(14,15)$. The use of some notional diagrams has even been considered to assess the efficacy of chemotherapy in prognostic prediction (13). However, to the best of our knowledge, no nomogram is available for the preoperative prediction of VI in CRC.

In the present study, a nomogram with clinical features for the individualized preoperative prediction of VI in patients with CRC was developed and validated. The goodness of fit, differentiation and clinical application value of the nomogram were evaluated. To the best of our knowledge, this is the first nomogram to predict preoperative VI in patients with CRC, which can provide preoperative optimization strategies for selected patients.

\section{Patients and methods}

Study population. The present retrospective analysis was approved by the Ethics and Human Subject Committee of Affiliated Tumor Hospital of Guangxi Medical University. According to the specific inclusion and exclusion criteria, the present study recruited 989 patients with CRC between August 2013 and April 2018 in the Affiliated Tumor Hospital of Guangxi Medical University. The inclusion criteria consisted of the following: i) Pathological confirmation of CRC in patients; ii) primary tumor resection had been performed; and iii) the status of VI was obtainable in the postoperative pathological report. The exclusion criteria included the following: i) Preoperative therapy involving radiotherapy, chemotherapy or chemoradiotherapy; ii) patients currently suffering from other cancer diseases; and iii) the presence of hereditary non-polyposis colon cancer or familial adenomatous polyposis. The corresponding demographic and preoperative clinical parameters, such as age, sex, body mass index (BMI), first-degree relatives' tumor history, blood routine examination, serum immunoglobulin level, tumor primary site, computed tomography (CT)-based on T stage (CT T stage) or $\mathrm{N}$ stage (CT N stage), preoperative histologic grade and tumor gross type, were collected. Weight change was obtained by self-reporting within the last three months prior to diagnosis and measured every week after hospitalization.

In total, 664 patients, including 389 male and 275 female patients, with complete information were enrolled. All 664 patients were randomly divided into two independent datasets at a ratio of 7:3 based on a computer-generated random number (training datasets: 468 cases; and validation datasets:
196 cases). $T$ and $\mathrm{N}$ stages were determined on the basis of the 7th edition of The American Joint Committee on Cancer, Cancer Staging Manual (16).

Feature selection. Least absolute shrinkage and selection operator (LASSO) is a penalized regression method that estimates the regression coefficients by maximizing the log-likelihood function, while restraining the sum of the absolute values of the regression coefficients (17). Regression coefficients estimated by LASSO are sparse, and many components are exactly 0 . Therefore, LASSO automatically deletes unnecessary covariates. The LASSO logistic regression algorithm is used for determining the regression of high-dimensional data, which is applied in many fields, including in genome-wide association studies, when it is difficult to find significant genetic factors with expected statistical significance in a large amount of data. The LASSO method can be used to screen out significant genetic factors with expected statistical significance, to produce a number of algorithms (18). The present study employed the LASSO logistic regression algorithm in the training dataset to select the most diagnostically predictive features. All of the categorical variables were transformed into dummy variables. The status of VI served as the dependent variable. The suitable tuning parameter $(\lambda)$ for LASSO logistic regression was determined using cross-validation. LASSO logistic regression was performed by package 'glmnet' function of 'glmnet' package. A minimum $\lambda$ was used for features selection. Features with non-zero coefficients at the optimal were selected by the LASSO logistic regression algorithm. Finally, the multiple logistic regression was performed using the diagnostic features selected by LASSO in the training dataset to construct the prediction model. The evaluation of the prediction model was performed in the validation dataset.

Nomogram construction and performance assessment. The prediction model was constructed in the training dataset, which used features selected by the LASSO algorithm, using a multivariate logistic regression model. All of the selected features entered the multivariate logistic regression model and the coefficient of each feature was calculated. The predicted index of each patient was calculated by the 'predict' function, based on the model constructed in the training dataset. A nomogram was formulated according to the resultants of the multivariable analyses, which incorporated the selected features. The goodness of fit between the observed value and the predicted value was examined by the calibration curve and tested using the Hosmer-Lemeshow test, which is a statistical test for goodness of fit for logistic regression models and used frequently in risk prediction models (19). An ideal calibration curve perfectly fits the 45 -degree reference line. The predictive discrimination of the nomogram was evaluated using the receiver operating characteristic (ROC) curve and the area under the curve (AUC). In the logistic regression model, the value of the AUC was the same as the concordance index (c-index). An AUC of 1.0 was determined, indicating perfect discrimination of the nomogram.

Validation of the nomogram. To assess the performance of the nomogram, the constructed nomogram was validated in the validation dataset. The predicted value of each patient in this 
dataset was calculated based on the formula constructed in the training dataset. The ROC and AUC were used to evaluate the predictive discrimination of the nomogram in the validation dataset. The calibration curve and Hosmer-Lemeshow test were used to assess the goodness of fit of the nomogram in the aforementioned dataset.

Decision curve analysis (DCA). The DCA method was employed to evaluate the clinical usefulness of the nomogram through quantitative training and verification, and compared with treat-all-patients scheme or the treat-none scheme to predict the net benefit under the dataset's different threshold probabilities (20). The treat-none scheme assumed no patient had a disease and the treat-all-patients scheme assumed all patients had a disease.

Statistical analysis. Statistical analysis was performed using R version 3.4.0 and RStudio (Version 1.1.447) (21,22). LASSO logistic regression analysis was carried out with the 'glmnet' software package (version 2.0-16; https://cran.r-project. org/web/packages/glmnet/index.html). In addition, the multivariate logistic regression analysis, nomogram building and calibration plots were conducted using the 'rms' package (version 5.1-3.1; https://cran.r-project.org/web/packages/rms/). The DCA and Hosmer-Lemeshow test were performed with the functions, 'dca.R' and 'HLtest.R,' respectively. $\mathrm{P}<0.05$ was considered to indicate a statistically significant difference.

\section{Results}

Clinical characteristics. A total of 664 patients were included in the analysis; $>200$ clinical parameters were collected in the present study. Patient demographics and pathologic parameters are listed in Table I. The average age of the included patients was 59.2 years (range, 17-87 years). In total, 314 patients had were diagnosed with rectal cancer, and 350 patients were diagnosed with colon cancer at the Affiliated Tumor Hospital of Guangxi Medical University. Of the 664 total patients, 139 had a history of first-degree relatives with tumors, while 525 patients did not. The average weight loss over the last 3 months was $1.73 \mathrm{kgk}$ (range, $-3-16 \mathrm{kgk}$ ). In addition, these patients were identified as VI-positive (203 cases) in the postoperative pathology report.

Feature selection. The LASSO logistic regression method was employed to select the most significant prediction features in the prediction model. The present study performed feature selection based on the training dataset. Overall, 154 features were used in the LASSO logistic regression. In addition, 4 features with non-zero coefficients were selected by the LASSO logistic regression algorithm with an optimal $\lambda$ of 0.048 (Fig. 1A and B). The 4 features included differentiation, CT-based N stage (CT N stage), hemameba and tumor distance from the anus $(\mathrm{cm})$.

Nomogram construction and performance assessment. The 4 features selected by LASSO logistic regression were included in the multivariate logistic regression modeling. As shown in Table II, multivariate logistic regression identified poor differentiation $\left(\mathrm{P}=4.66 \times 10^{-3}\right)$, CT-based $\mathrm{N} 1 / \mathrm{N} 2$ stage
$\left(\mathrm{P}=4.48 \times 10^{-6}\right)$ and hemameba $(\mathrm{P}=0.02)$ as independent impact factors of VI. The present study constructed a nomogram based on the features (Fig. 2). A nomogramn can indicate the points assigned to each variable by the top of the scale. The summation of each point (the total points) can be transformed to predict the possible risk of VI for patients at the lowest scale.

The calibration plot of the nomogram for the probability of VI demonstrated a good agreement between prediction and observation in the training data set (Fig. 3A). The P-value for the Hosmer-Lemeshow test was 0.60 (Fig. 3A), which indicated that there was no departure from a perfect fit. The AUC for the prediction nomogram was 0.731 in the training dataset (Fig. 3B)

These 4 features were used in the construction of the nomogram (Fig. 2). Each feature corresponds to a specific point by drawing a straight line up to the point axis. After the summation of the points has been plotted on the master axis, which represents the probability of VI, it is drawn directly down to the diagnostic axis. For example, 1 patient with CRC was recorded to have poor differentiation (100 points), a CT N stage of N1 (32 points), a tumor distance from the anus $<5 \mathrm{~cm}$ (10 points), and the hemameba count was $10 \times 10^{9}$ (51 points). In this example, the total points equated to equals 193 , and the VI probability is $\sim 60 \%$. According to the $50 \%$ threshold for this patient, they are high-risk and neoadjuvant chemotherapy should be considered.

Validation of the nomogram. The present study observed good identification (Fig. 4B) and good calibration (Fig. 4A) in the validation dataset. The nomogram exhibited an AUC of 0.710 (Fig. 4B). Validation of the calibration curve exhibited good concordance between the predicted probability and actual probability. The Hosmer-Lemeshow test yielded a non-significant statistic ( $\mathrm{P}=0.281$; Fig. $4 \mathrm{~A})$.

Clinical usefulness of the nomogram. Predicted probability of VI could be obtained from the nomogram. With the DCA based on 664 patients, the present study performed decision-making based on the evaluation for improvement of the nomogram. As shown in Fig. 5, the DCA curve indicated that if the probability of producing VI by the nomogram is $>20$ and $<70 \%$, it is more beneficial to predict VI with the treat-all-patients scheme or the treat-none scheme. For example, with a $60 \%$ probability of VI, the nomogram increases the net benefit by $4.4 \%$ of the treat-all-patients scheme or the treat-none scheme. This suggests the nomogram is clinically useful.

\section{Discussion}

In the present study, the single preoperative clinical features of a nomogram for the prediction of VI in patients with CRC, combined with clinical features was constructed and validated. Nomograms have high prediction accuracy and reliability. To the best of our knowledge, this is the first preoperative predictive tool for patients with CRC, who are at a high-risk of VI, in addition to facilitating the preoperative optimization strategy for this group. Although magnetic resonance imaging (MRI) and $\mathrm{CT}$ are the main diagnostic methods recommended by the NCCN guidelines for preoperative clinical staging, they are also the main diagnostic basis for the differential diagnosis of VI. 
Table I. Patient characteristics.

\begin{tabular}{|c|c|c|c|c|c|}
\hline Factor & $\mathrm{n}$ & $\%$ & Factor & $\mathrm{n}$ & $\%$ \\
\hline Age, years & & & Vascular invasion & & \\
\hline $17-30$ & 14 & 2.1 & No & 461 & 69.4 \\
\hline $31-45$ & 69 & 10.4 & Yes & 203 & 30.6 \\
\hline $46-60$ & 259 & 39.0 & Lymphovascular invasion & & \\
\hline$>60$ & 322 & 48.5 & No & 429 & 64.6 \\
\hline Sex & & & Yes & 235 & 35.4 \\
\hline Male & 389 & 58.6 & & & \\
\hline Female & 275 & 41.4 & CT, computed tomography. & & \\
\hline
\end{tabular}

Body mass index, $\mathrm{kg} / \mathrm{m}^{2}$

$\leq 18.4$

73

18.5-23.9

24-27.9

$\geq 28$

431

136

24

Primary site

Rectum

Colon

314

350

Weight loss, kg

$<3$

474

3-6

132

$>6$

First-degree relatives' tumor history

No

Yes

CT T Stage

$\mathrm{T} 1$

T2

T3

T4

CT N Stage

No

N1

$\mathrm{N} 2$

Differentiation

Well

Moderately

Poorly

Tumor gross type

Ulceration

Infiltrative

Ulceration and Infiltrative

Protruded

Other

Tumor distance from anus, $\mathrm{cm}$

$<5$

5-10

11-15

$>15$

Perineural invasion

No

Yes
11.0

Table I. Continued.

64.9

20.5

3.6

Table II. Multivariable logistic regression analysis of the selected clinical features in the training set.

\begin{tabular}{lcc}
\hline Variable & Odds ratio $(95 \% \mathrm{CI})$ & P-value \\
\hline $\begin{array}{l}\text { Differentiation } \\
\text { Well }\end{array}$ & 1 & \\
Moderately & $5.92(1.17-108.09)$ & 0.09 \\
Poorly & $20.52(3.77-384.19)$ & $4.66 \times 10^{-3}$ \\
CT N Stage & & \\
N0 & 1 & \\
N1/N2 & & $4.48 \times 10^{-6}$ \\
Tumor distance & $2.73(1.78-4.22)$ & \\
from anus, cm & & \\
$<5$ & $0.76(0.34-1.73)$ & 0.50 \\
$5-10$ & $1.95(0.81-4.81)$ & 0.14 \\
$11-15$ & $0.88(0.42-1.89)$ & 0.74 \\
$>15$ & $0.88(0.79-0.97)$ & 0.02 \\
Hemameba & & \\
\hline
\end{tabular}

55.9

28.6

15.5

CT N Stage, computed tomography-based N stage; CI, confidence interval.
3.8

80.9

15.4

50.8

6.5

6.0

36.0

0.8

9.8

24.8

11.0

54.4
However, due to the limitations of these imaging techniques, CT and MRI need to accurately identify VI, especially small vessel invasion; however, technical problems remain, affecting the staging and prognosis $(23,24)$. A previous reported that the accuracy of CT recognition of VI is $30.9 \%$, and the accuracy of MRI recognition of VI is $54 \%(23,24)$. However, the accuracy of CT and MRI in the identification of VI is associated with the clinical experience of doctors, therefore this accuracy rate may be even lower $(23,24)$. However, the present nomogram revealed that the AUC value of VI is 0.731 , which is of high sensitivity and specificity. Both have their own advantages, however the combination of the two can better identify VI $(23,24)$. VI is a fundamental determinant of solid tumor progression, which is a strong prognostic indicator in CRC (25). A number of studies have demonstrated that VI is a negative prognostic index for the survival of patients undergoing radical resection of CRC $(3,9,16)$.

The tumor microenvironment (TME) is a complex system composed of cells, cytokines and extracellular matrix (26). VI 

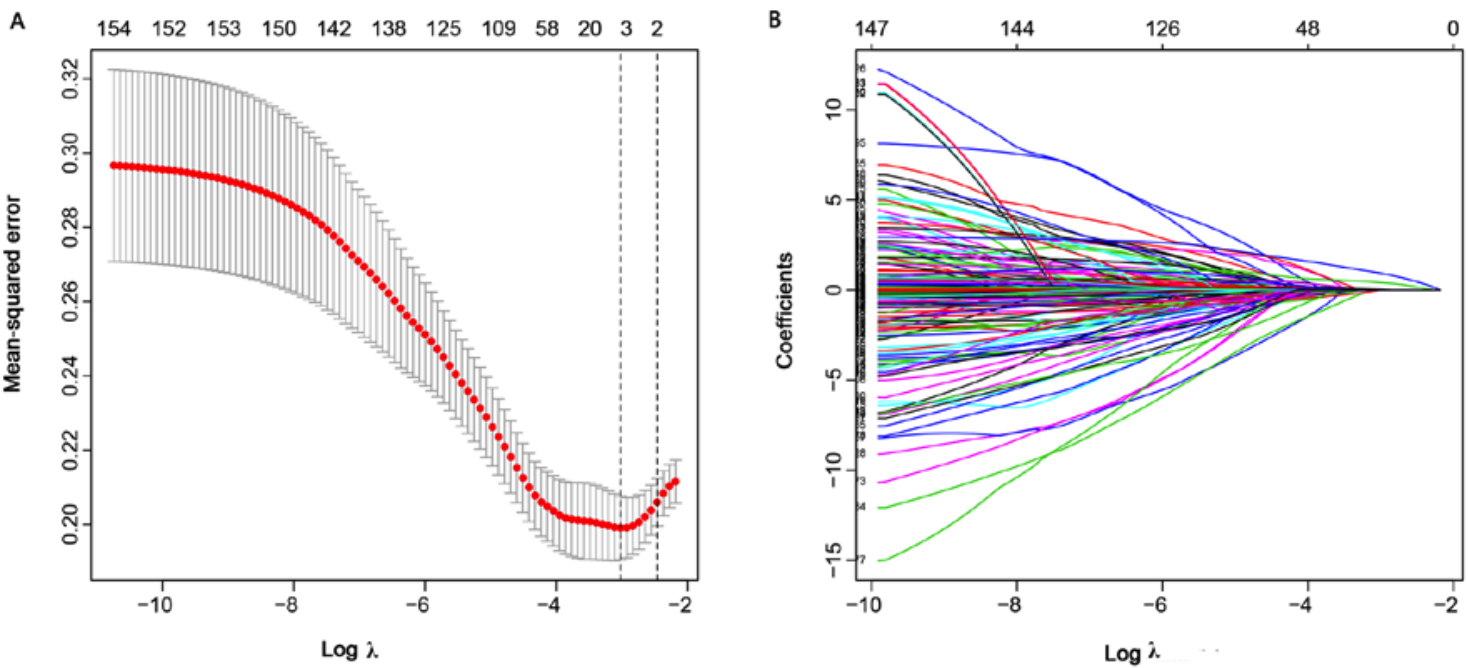

Figure 1. Texture feature selection using the LASSO binary logistic regression model. (A) By selecting a 10 -fold cross-validation in the LASSO model with minimum standards. The binomial deviance was plotted versus $\log (\lambda)$. Dotted vertical lines were drawn at the optimal $\lambda$ values based on the minimum criteria and 1 standard error of the minimum standards and the optimal $\lambda$ was 0.048 .(B) The LASSO logistic regression algorithm was used to screen out 4 features with non-zero coefficients out of 154 features. LASSO, least absolute shrinkage and selection operator.

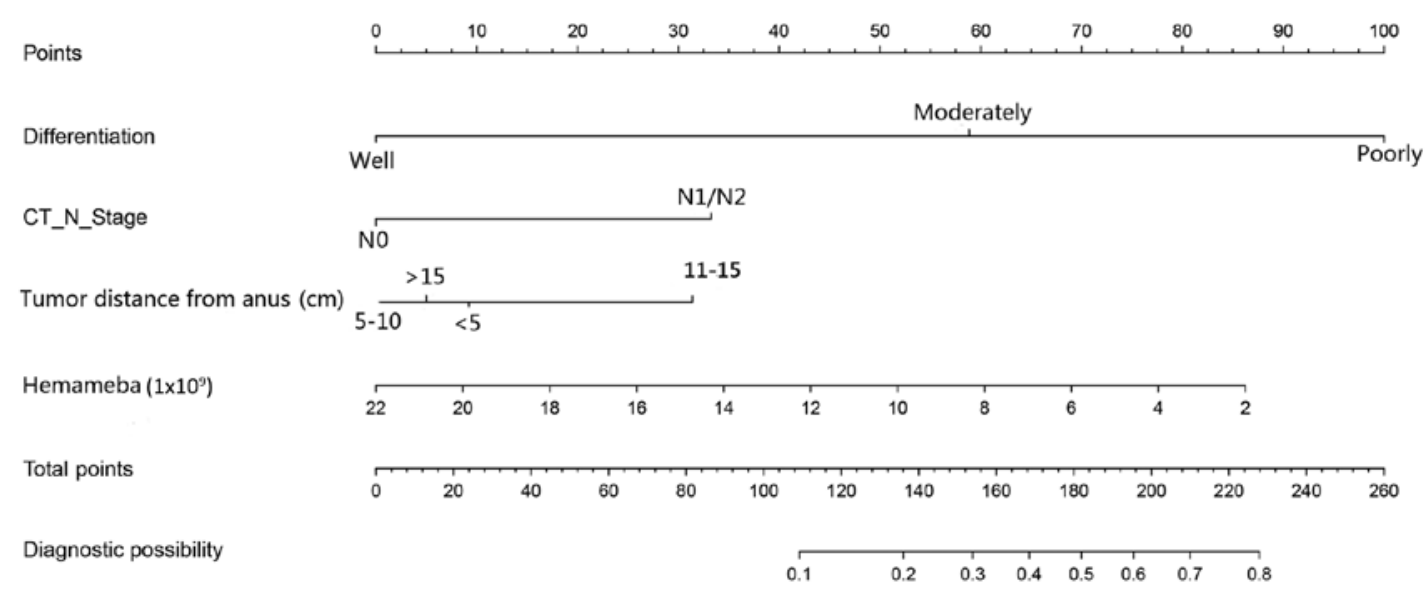

Figure 2. Developed clinical features nomogram, with the features: Differentiation, CT N Stage, tumor distance from anus (cm) and hemameba. CT N Stage, computed tomography-based $\mathrm{N}$ stage.
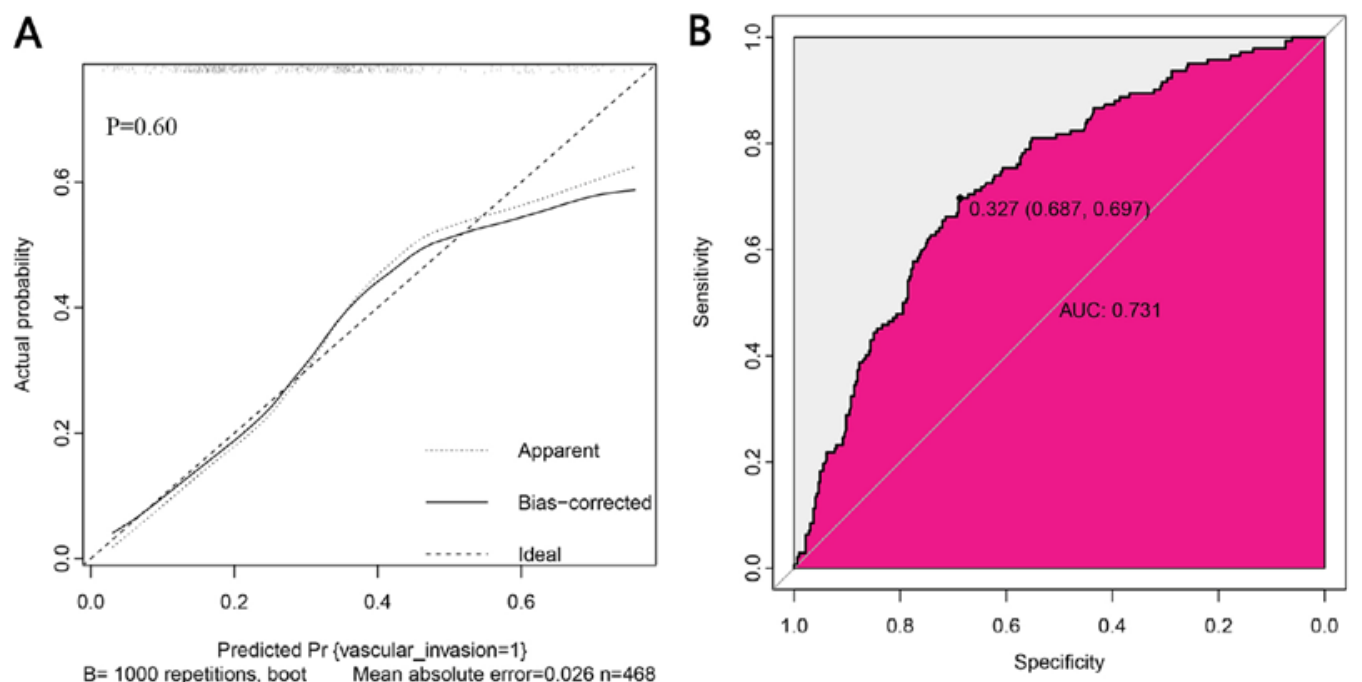

Figure 3. The performance of the nomogram in the training set. The LASSO algorithm and the Hosmer-Lemeswell test was used in the training set. (A) Calibration curve of the nomogram in the training dataset. (B) AUC curve of the nomogram in the training dataset. AUC, area under the curve; LASSO, least absolute shrinkage and selection operator. 
A

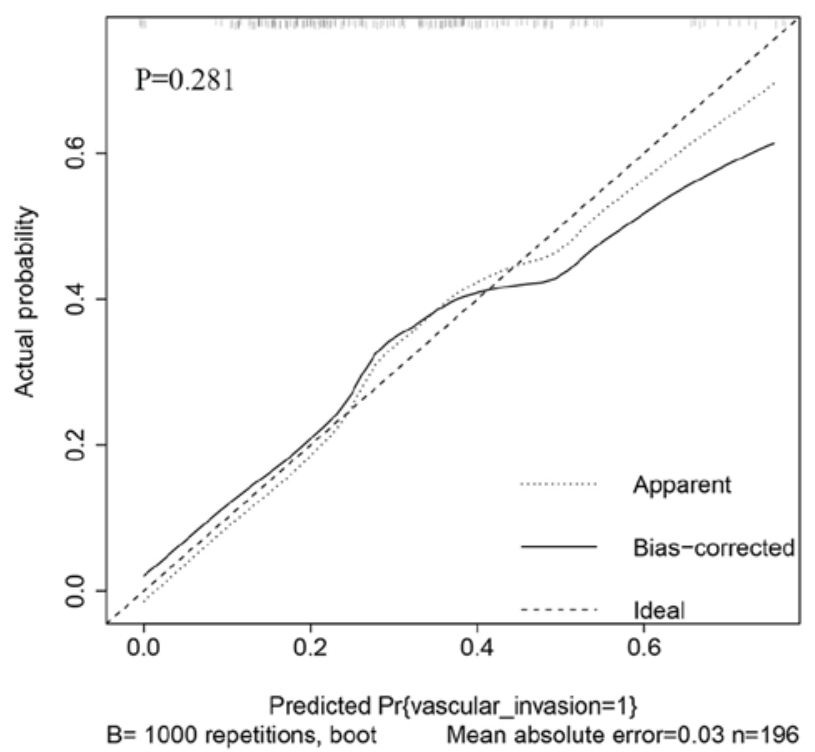

B

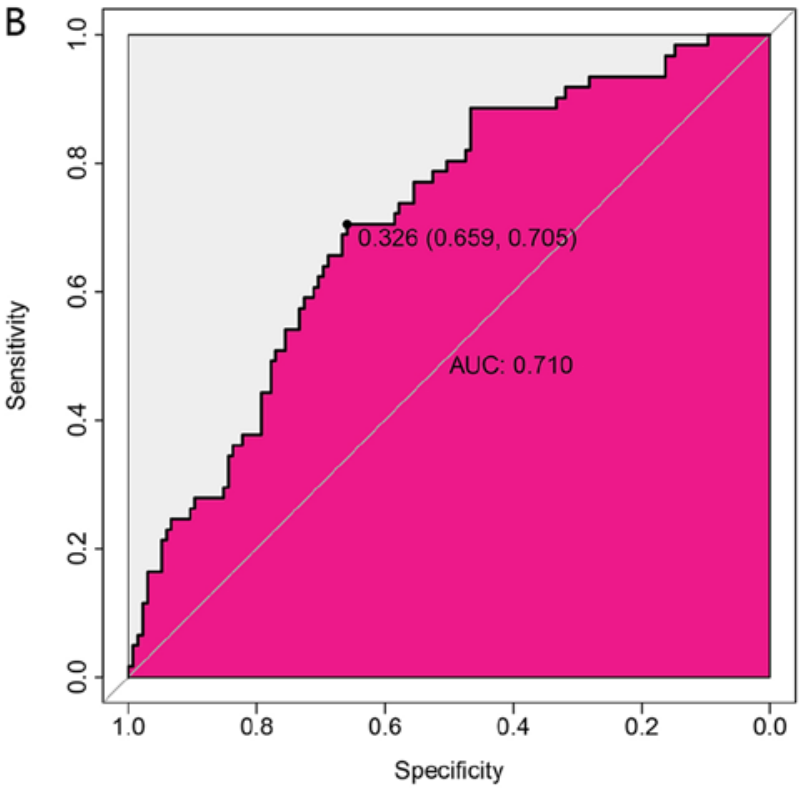

Figure 4. The performance of the nomogram in the validation set. The LASSO algorithm and the Hosmer-Lemeswell test was used in the validation set. (A) Calibration curve of the nomogram in the validation set. (B) AUC curve of the nomogram in the validation set. AUC, area under the curve; LASSO, least absolute shrinkage and selection operator.

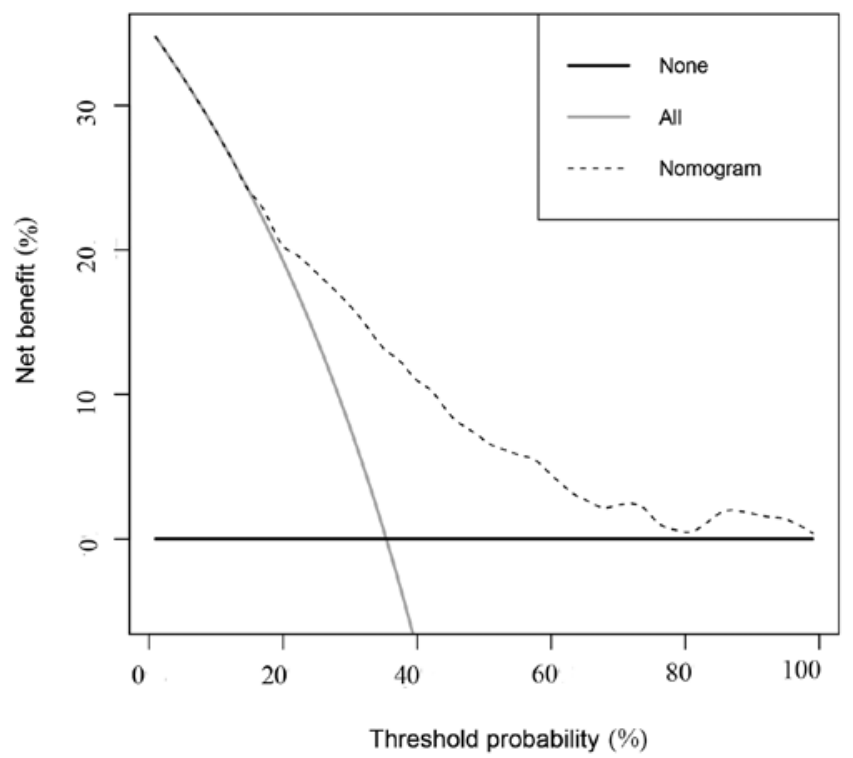

Figure 5. DCA for the VI production nomogram and the model. The y-axis shows the net benefit. The dotted line represents the VI production nomogram. The grey line represents the assumption that all patients have VI. The thin black line represents the assumption that no patients have VI. When the nomogram is $>20$ and $<70 \%$, the dotted line indicates that patients benefit from the nomogram. DCA, decision curve analysis; VI, vascular invasion.

is associated with the influence of TME $(26,27)$. Tumor cell proliferation requires nutrients and energy, and vessels are important channels for providing the above (27). The secretion of VEGF, fibroblast growth factor, angiopoietin-like proteins and the corresponding inflammatory cells by tumor cells and other cells in the TME environment can promote the formation of new vessels $(28,29)$. This can lead to the recurrence and metastasis of the tumor (27-29). Therefore, VI-positive can mean the progression or recurrence of the disease (30).
To construct a nomogram for the preoperative prediction of VI in patients with CRC, the present study first screened out the most significant predictive features using a LASSO logistic regression. This method has been widely used in the feature selection of high-dimensional data (18). It is more suitable than the linear regression method to analyze the data of the selection study with dichotomous fitness results, and it is regarded as an analytical tool for the empirical study of multivariate selection (31).

The nomogram had favorable discrimination and calibration, with a P-value for the Hosmer-Lemeshow test of 0.60 and an AUC of 0.731 in the training dataset. The results of the nomogram were evaluated in the validation dataset, which indicated that the nomogram had reasonable discrimination and good calibration, with an AUC of 0.710 and a P-value 0.281 via the Hosmer-Lemeshow test. The DCA curve showed that if the probability of VI generated by the nomogram was $>20$ and $<70 \%$, the prediction of VI will be more effective than either the treat-all scheme or the treat-none scheme. Therefore, the preoperative nomogram could be used as a clinical predictor of VI in patients with CRC.

A total of 4 factors were finally identified by the present nomogram: Differentiation, CT N stage, hemameba and tumor distance from the anus. Previous reports have demonstrated that these factors have an unusual impact on the prognosis of CRC (32-34), however, to the best of our knowledge, this is the first time they have been incorporated for modeling. A previous study reported the association between VI and distant metastasis and depth of invasion (32). As the differentiation level is associated with the malignant degree of the tumor, this study suggests that the degree of differentiation may be a reasonable predictor of VI $(3,32)$. Preoperative MRI examination is an effective and convenient method for evaluating tumor staging, depth of invasion and local metastasis (35). 
Inflammatory markers include inflammatory cells, various immune cells, cytokines, chemokines and pro-inflammatory mediators, of which hemameba is the most significant indicator $(28,36)$. This is because hemameba is associated with the pathogenesis of various inflammatory processes, including allergies, parasitic diseases, bacterial and viral infections, and tumor immune tissue damage $(29,33,37)$. Chronic inflammation can be caused by tumor proliferation (34). Hemameba accumulate in inflammatory sites through blood circulation (28). Hemameba and their secreted products promote the development and metastasis of tumors through the immune response. For example, CCL 20/CCR 6 mediate organ selective liver metastasis of colorectal cancer (29,36-39).

A previous tudy reported that $\sim 20 \%$ of cancer-related mortality is associated with inflammatory cells leading to cell transformation and the enhancement of tumor cell invasion. White blood cells are an important component of inflammatory cells (27). Hemameba also play an important role at different stages of tumor development, including initiation, promotion, malignant conversion, invasion and metastasis (34), which is the index of tumor therapy. It can also be used to predict postoperative infection in patients (40). A previous study also reported that it is an independent risk factor for surgical site infection (40).

The present study presented with several limitations. First of all, due to the single center utilized for retrospective research, potential selection bias is inevitable. Secondly, although a genome classifier is a promising predictor, no application of genome features has been considered. In addition, the sample size of the validation set is small, which may affect the credibility of the evaluation results to some extent. Finally, the validation of the results using the same cohort of patients is another potential limitation of the study, and a larger external validation with multi-center and larger samples may the optimal choice. Therefore, further efforts are required to collect additional data and incorporate more impartial predictors to improve the performance of the model.

In conclusion, this model has good discrimination and calibration capability. It may be used in patients with CRC prior to surgery as it predicted VI for the majority of patients and it may help to provide accurate treatment options.

\section{Acknowledgements}

Not applicable.

\section{Funding}

The present study was supported by the Self-financing Research Project of the Health and Family Planning Commission of Guangxi Zhuang Autonomous Region (grant no. Z2015607), Guangxi Medical and Health Appropriate Technology Development and Promotion Application Project (grant no. S2017098) and Guangxi Science and Technology Department Project (grant no. GuikeAB16380202).

\section{Availability of data and materials}

The datasets used and/or analyzed in the present study are available from the corresponding author on reasonable request.

\section{Authors' contributions}

WT, LG, WX, XH and JL conceived and designed the experiments. XH, JL, GW, FJ, SC, CZ, WX, WT, LG, WY, CL and $\mathrm{ZL}$ performed the experiments. XH, JL and $\mathrm{GW}$ analyzed the data. XH, JL, GW, FJ, SC, CZ, WX, WT, WY, CL and ZL contributed to the reagents, materials and analysis tools used in the present study. XH, JL, WT and WX wrote the manuscript. All authors have read and approved the final manuscript.

\section{Ethics approval and consent to participate}

This study was approved by the Ethics and Human Subject Committee of Affiliated Tumor Hospital of Guangxi Medical University (approval no. LW2019020, Nanning, China). Due to the retrospective design of the current study and patient anonymization, the review board determined that informed consent was not required.

\section{Patient consent for publication}

Not applicable.

\section{Competing interests}

The authors declare that they have no competing interests.

\section{References}

1. Bray F, Ferlay J, Soerjomataram I, Siegel RL, Torre LA and Jemal A: Global cancer statistics 2018: GLOBOCAN estimates of incidence and mortality worldwide for 36 cancers in 185 countries. CA Cancer J Clin 68: 394-424, 2018.

2. Siegel RL, Miller KD and Jemal A: Cancer statistics, 2019. CA Cancer J Clin 69: 7-34, 2019.

3. Benson AB III, Venook AP, Cederquist L, Chan E, Chen YJ, Cooper HS, Deming D, Engstrom PF, Enzinger PC, Fichera A, et al: Colon cancer, version 1.2017, NCCN clinical practice guidelines in oncology. J Natl Compr Canc Netw 15: 370-398, 2017.

4. Betge J, Pollheimer MJ, Lindtner RA, Kornprat P, Schlemmer A, Rehak P, Vieth M, Hoefler G and Langner C: Intramural and extramural vascular invasion in colorectal cancer: Prognostic significance and quality of pathology reporting. Cancer 118: 628-138, 2012.

5. Siddiqui MRS, Simillis C, Hunter C, Chand M, Bhoday J, Garant A, Vuong T, Artho G, Rasheed S and Tekkis P: A meta-analysis comparing the risk of metastases in patients with rectal cancer and MRI-detected extramural vascular invasion (mrEMVI) vs mrEMVI-negative cases. Br J Cancer 116: 1513-1519, 2017.

6. Blumberg D,Paty PB,Picon AI, Guillem JG,Klimstra DS, Minsky BD, Quan SH and Cohen AM: Stage I rectal cancer: Identification of high-risk patients. J Am Coll Surg 186: 574-580, 1998.

7. Bentzen SM, Balslev I, Pedersen M, Teglbjaerg PS, Hanberg-Sørensen F, Bone J, Jacobsen NO, Sell A, Overgaard J, Bertelsen K, et al: Time to loco-regional recurrence after resection of Dukes' B and C colorectal cancer with or without adjuvant postoperative radiotherapy. A multivariate regression analysis. Br J Cancer 65: 102-107, 1992.

8. Bhangu A, Fitzgerald JE, Slesser A, Northover JM, Faiz O and Tekkis P: Prognostic significance of extramural vascular invasion in T4 rectal cancer. Colorectal Dis 15: e665-e671, 2013.

9. Benso AB, Venook AP, Al-Hawary MM, Cederquist L, Chen YJ, Ciombor KK, Cohen S, Cooper HS, Deming D and Engstrom PF: NCCN guidelines insights: Colon cancer, version 2.2018. J Natl Compr Canc Netw 16: 359-369, 2018.

10. Kaipainen A, Korhonen J, Pajusola K, Aprelikova O, Persico MG, Terman BI and Alitalo K: The related FLT4, FLT1, and KDR receptor tyrosine kinases show distinct expression patterns in human fetal endothelial cells. J Exp Med 178: 2077-2088, 1993. 
11. Nguyen QD, Rodrigues S, Rodrigue CM, Rivat C, Grijelmo C Bruyneel E, Emami S, Attoub S and Gespach C: Inhibition of vascular endothelial growth factor (VEGF)-165 and semaphorin 3A-mediated cellular invasion and tumor growth by the VEGF signaling inhibitor ZD4190 in human colon cancer cells and xenografts. Mol Cancer Ther 5: 2070-2077, 2006.

12. Downs AW: Blood, A study in general physiology. Can Med Assoc J 19: 754, 756, 1928

13. Massacesi C, Norman A, Price T, Hill M, Ross P and Cunningham D: A clinical nomogram for predicting long-term survival in advanced colorectal cancer. Eur J Cancer 36 : 2044-2052, 2000.

14. Zhou Q, Wu ZY and Lin ZQ: A nomogram to predict prognosis in Ewing sarcoma of bone. J Bone Oncol 15: 100223, 2019.

15. Zhao M, Yin J, Yang X, Jiang T, Lu T, Huang Y, Li M, Yang X, Lin M, Niu H, et al: Nomogram to predict thymoma prognosis: A population-based study of 1312 cases. Thorac Cancer 10: $1167-1175,2019$.

16. Edge SB and Compton CC: The American Joint Committee on cancer: The 7th edition of the AJCC cancer staging manual and the future of TNM. Ann Surg Oncol 17: 1471-1474, 2010

17. Bickel PJ, Ritov Y and Tsybakov AB: Simultaneous analysis of Lasso and dantzig selector. Ann Stat 37: 1705-1732, 2009.

18. Jiang $\mathrm{Y}, \mathrm{He} \mathrm{Y}$ and Zhang $\mathrm{H}$ : Variable selection with prior information for generalized linear models via the prior LASSO method. J Am Stat Assoc 111: 355-376, 2016.

19. Paul P, Pennell ML and Lemeshow S: Standardizing the power of the Hosmer-Lemeshow goodness of fit test in large data sets. Stat Med 32: 67-80, 2013

20. Van Calster B, Wynants L, Verbeek JFM, Verbakel JY, Christodoulou E, Vickers AJ, Roobol MJ and Steyerberg EW: Reporting and interpreting decision curve analysis: A guide for investigators. Eur Urol 74: 796-804, 2018.

21. R Core Team. R: A language and environment for statistical computing. R Foundation for Statistical Computing, Vienna, Austria, 2012. ISBN 3-900051-07-0, URL http://www.R-project. org/.

22. RStudio Team. RStudio: Integrated development for R. RStudio, Inc, Boston, MA, 2015. URL http://www.rstudio.com/.

23. Jhaveri KS, Hosseini-Nik H, Thipphavong S, Assarzadegan N, Menezes RJ, Kennedy ED and Kirsch R: MRI Detection of extramural venous invasion in rectal cancer: Correlation with histopathology using elastin stain. AJR Am J Roentgenol 206 : $747-755,2016$

24. Yao X, Yang SX, Song XH, Cui YC, Ye YJ and Wang Y: Prognostic significance of computed tomography-detected extramural vascular invasion in colon cancer. World J Gastroenterol 22: $7157-7165,2016$

25. Angelucci A, Delle Monache S, Cortellini A, Di Padova M and Ficorella C: 'Vessels in the Storm': Searching for prognostic and predictive angiogenic factors in colorectal cancer. Int $\mathrm{J}$ Mol Sci 19: pii: E299, 2018.

26. Egeblad M, Nakasone ES and Werb Z: Tumors as organs: Complex tissues that interface with the entire organism. Dev Cell 18: 884-901, 2010.
27. Bahrami A, Khazaei M, Hassanian SM, ShahidSales S, Joudi-Mashhad M, Maftouh M, Jazayeri MH, Parizade MR, Ferns GA and Avan A: Targeting the tumor microenvironment as a potential therapeutic approach in colorectal cancer: Rational and progress. J Cell Physiol 233: 2928-2936, 2018.

28. Carbone C, Piro G, Merz V, Simionato F, Santoro R, Zecchetto C, Tortora G and Melisi D: Angiopoietin-like proteins in angiogenesis, inflammation and cancer. Int J Mol Sci 19: pii: E431, 2018.

29. Kounis NG, Soufras GD, Tsigkas G and Hahalis G: White blood cell counts, leukocyte ratios, and eosinophils as inflammatory markers in patients with coronary artery disease. Clin Appl Thromb Hemost 21: 139-143, 2015.

30. Courtney ED, West NJ, Kaur C, Ho J, Kalber B, Hagger R, Finlayson $\mathrm{C}$ and Leicester RJ: Extramural vascular invasion is an adverse prognostic indicator of survival in patients with colorectal cancer. Colorectal Dis 11: 150-156, 2009.

31. Janzen FJ and Stern HS: Logistic regression for empirical studies of multivariate selection. Evolution 52: 1564-1571, 1998.

32. Fujii T, Sutoh T, Morita H, Yajima R, Yamaguchi S, Tsutsumi S, Asao $\mathrm{T}$ and Kuwano H: Vascular invasion, but not lymphatic invasion, of the primary tumor is a strong prognostic factor in patients with colorectal cancer. Anticancer Res 34: 3147-3151, 2014.

33. Shalapour S and Karin M: Immunity, inflammation, and cancer: An eternal fight between good and evil. J Clin Invest 125: 3347-3355, 2015.

34. Singh R, Mishra MK and Aggarwal H: Inflammation, immunity, and cancer. Mediators Inflamm 2017: 6027305, 2017.

35. Elmi A, Hedgire SS, Covarrubias D, Abtahi SM, Hahn PF and Harisinghani M: Apparent diffusion coefficient as a non-invasive predictor of treatment response and recurrence in locally advanced rectal cancer. Clin Radiol 68: e524-e531, 2013.

36. Huang WY, Berndt SI, Shiels MS, Katki HA, Chaturvedi AK, Wentzensen N, Trabert B, Kemp TJ, Pinto LA, Hildesheim A, et al: Circulating inflammation markers and colorectal adenoma risk. Carcinogenesis 40: 765-770, 2019.

37. Oliver-Baxter JM, Whitford HS, Turnbull DA and Bond MJ: Effects of vitamin supplementation on inflammatory markers and psychological wellbeing among distressed women: A randomized controlled trial. J Integr Med 16: 322-328, 2018.

38. Qian BZ and Pollard JW: Macrophage diversity enhances tumor progression and metastasis. Cell 141: 39-51, 2010.

39. Lin WF, Lu JY, Cheng BB and Ling CQ: Progress in research on the effects of traditional Chinese medicine on the tumor microenvironment. J Integr Med 15: 282-287, 2017.

40. Sagawa M, Yoshimatsu K, Yokomizo H, Yano Y, Okayama S, Usui T, Yamaguchi K, Shiozawa S, Shimakawa T, Katsube T, et al: Worse preoperative status based on inflammation and host immunity is a risk factor for surgical site infections in colorectal cancer surgery. J Nippon Med Sch 84: 224-230, 2017.

This work is licensed under a Creative Commons Attribution-NonCommercial-NoDerivatives 4.0 International (CC BY-NC-ND 4.0) License. 